\title{
Hand gesture based dumb and deaf communication using smart gloves
}

\author{
Sneha $T^{1}$, Sushma Desai ${ }^{2}$, Sahana $M^{3}$ \\ ${ }^{1}$ B.E. $8^{\text {th }}$ sem, Department of computer science and engineering, \\ RYMEC, Ballari, Visvesvaraya Technological University, \\ ${ }^{2}$ B.E. $8^{\text {th }}$ sem, Department of computer science and engineering, \\ RYMEC, Ballari, Visvesvaraya Technological University, \\ ${ }^{3}$ B.E. $8^{\text {th }}$ sem, Department of computer science and engineering, \\ RYMEC, Ballari, Visvesvaraya Technological University,
}

Abstract: The main aim of this paper is to provide voice to voiceless people with the help of Smart Gloves. With the proposed work, sign language is converted into speech using accelerometer and microcontrollers. where it reduces the communication barrier between two communities using the smart gloves.

Keywords: Sign language, smart gloves, accelerometer sensor, microcontroller.

\section{Introduction}

Communication is a process of exchanging ideas, thoughts, feelings and information in form of verbal or non verbal message. According to recent survey in the world 7.6 million of the total population suffers from deafness and dumbness. These people faces difficulties to convey there messages within the society. The sign language is an important and only method of communication for deaf-dumb persons, but most of the time the normal people find it difficult to understand this sign language. Using sign language for each sign the respective voice output is obtained via MP3 module and text message is obtained using LCD display additionally those people can operate home appliance like fan and lights through hand gesture movement.

\section{Existing system}

Vision based methods can be used without any restrictions upon the user. Hand tracking Is vital segment for gesture recognition, in which the signer's or user's hands must be detected and localized in image frames. Where detection requires proper lightening if not the gesture cannot be captured accurately.

\section{3. proposed system}

The proposed system aims to give voice to voiceless people with the help of smart gloves. In this the accelerometer are used for sign language for each sign the respective voice output is obtained via MP3 module text message is obtained by LCD display through hand gesture movement. Tilting of the palm can be captured by the accelerometer sensors can measure the bend of the five fingers when making a sign. For each sign the voice will be recorded and stored in $\mathrm{Sd}$ card. When the user performs a gesture the signs are captured by the microcontroller which converts the analog signals to digital values through its 8-channel ADC.

\section{Accelerometer}

Accelerometer sensor senses the gesture made by the user these sensor gives the output in the form of voltage variation that varies with degree of bend. This output is then sent to the ADC channel of microcontroller.

\section{ADC channel of microcontroller}

This microcontroller processes the signals and performs anlog to digital convertion further the data is sent in wireless manner to the receiver section. In this section the gesture is recognized and corresponding output is displayed on the LCD and 
simultaneously speech output is playback through speaker.

\section{LCD(liquid crystal display)}

LCD is a flat panel display, electronic visual display, based on Liquid Crystal Technology liquid crystal display consists of an array of tiny segments (called pixels) that can be manipulated to present information.

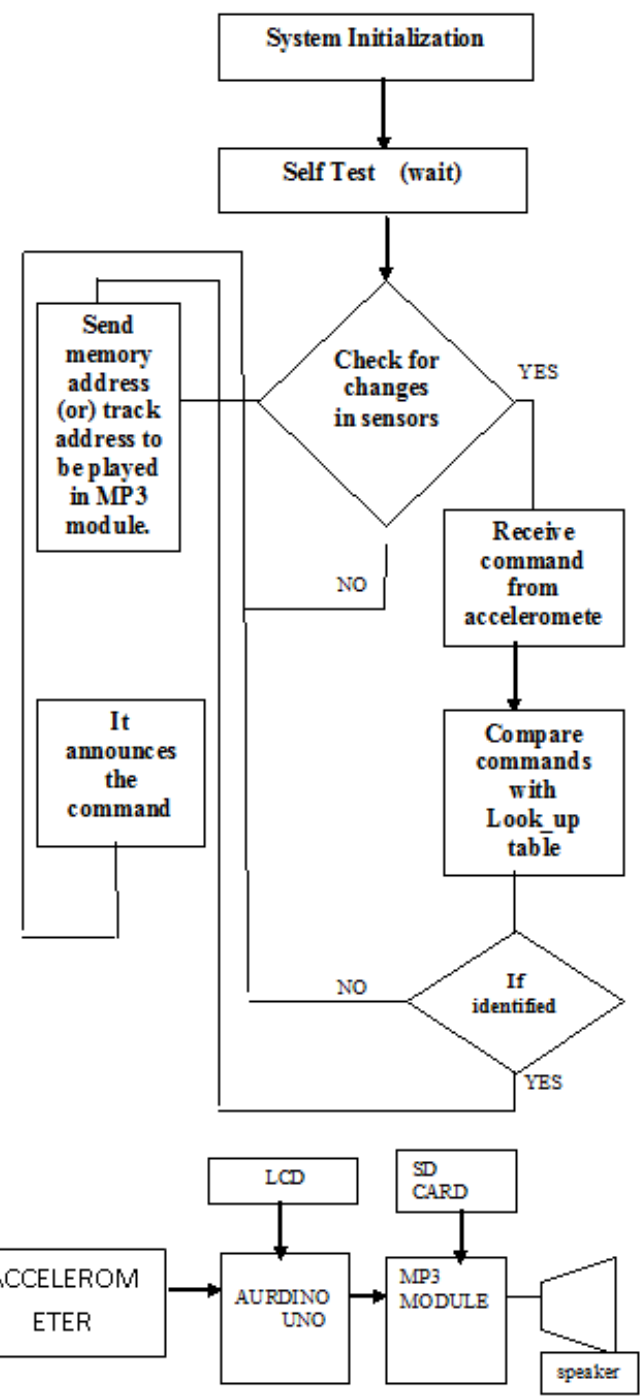

Fig: (Block diagram for the sign language detection and recognition system for dumb and deaf people)

\section{Conclusion}

This project describes the design and working of a system which is useful for dumb and deaf to communicate with one another and with the normal people. The dumb people use their standard sign language which is not easily understandable by common people and feels difficult to understand their gestures. This system converts the sign language into voice which is easily understandable by normal people. The sign language is translated into text form and displayed on LCD to facilitate the deaf people as well.

\section{Future Work}

Going further, most of the units can be embedded along with the controller on a single board with change in technology, thereby reducing the size of the system. and making more user friendly and portable

\section{References}

[1] Andrew Wilson and Aaron Bobick, "Learning visual behavior for gesture analysis," In Proceedings of the IEEE Symposium on Computer Vision, Coral Gables, Florida, pp. 1921, November 1995.

[2] Ginu Thomas, "A Review of Various Hand Gesture Recognition Techniques," VSRDIJEECE, Vol. 1 (7), 2011,pp. 374-383.

[3] Surachai, Stewart, Ahmet, "Two Hand Tracking using Color Statistical Model with the Kmeans Embedded Particle Filter for Hand Gesture Recognition", 7th Computer Information Systems and Industrial Management Applications, 2008, pp. 201-205.

[4] Pham, Nguyen, TuKhoa, "A New Approach to Hand Tracking and Gesture Recognition by a New Feature Type and HMM", Sixth International Conference on Fuzzy Systems and Knowledge Discovery, IEEE Computer Society, 2009, pp. 3-6. 